\title{
Upregulation of microfibrillar-associated protein 2 is closely associated with tumor angiogenesis and poor prognosis in hepatocellular carcinoma
}

\author{
NU ZHANG, FENG SHAO and WEIDONG JIA \\ Department of General Surgery, Anhui Provincial Hospital, Cheeloo College of Medicine, \\ Shandong University, Jinan, Shandong 250021, P.R. China
}

Received March 2, 2021; Accepted July 12, 2021

DOI: $10.3892 / \mathrm{ol} .2021 .13000$

\begin{abstract}
Abnormal expression of microfibrillar-associated protein 2 (MFAP2), a key regulator of cellular differentiation, affects the occurrence and progression of tumors. However, the underlying role of MAFP2 in hepatocellular carcinoma (HCC) remains unclear. In the present study, patterns of MFAP2 expression in HCC were analyzed using sequencing data from The Cancer Genome Atlas database. Expression profiles of MFAP2, as well as those of epithelial-mesenchymal transition (EMT)-related proteins, were compared between HCC pathological sections and fresh tissues. Thereafter, associations between patterns of MFAP2 expression and the clinicopathological characteristics of patients, and identified risk factors associated with disease-free survival (DFS) and overall survival (OS), were determined. The functions of MFAP2 in the EMT-induced proliferation and migration of MHCC $97 \mathrm{H}$ cells were investigated using in vitro experiments, and the effects of MFAP2 on vascular endothelial growth factor A (VEGFA)-induced tumor angiogenesis were also investigated. Upregulation of MFAP2 expression was observed in HCC, and was often accompanied by the abnormal expression of EMT-related marker proteins. In addition, analysis of clinical data from 94 patients with tumor tissues revealed a significant positive correlation between MFAP2 expression and low DFS and low OS following surgery. Through in vitro experimentation, silencing MFAP2 expression was shown inhibit EMT, which thereby inhibited cellular proliferation and migration. Moreover, downregulation of MFAP2 inhibited tumor angiogenesis via the inhibition of VEGFA. Taken
\end{abstract}

Correspondence to: Professor Weidong Jia, Department of General Surgery, Anhui Provincial Hospital, Cheeloo College of Medicine, Shandong University, 44 Wen Hua Xi Road, Jinan, Shandong 250021, P.R. China

E-mail: jiaweidong901@163.com

Key words: microfibrillar-associated protein 2, hepatocellular carcinoma, epithelial-mesenchymal transition, biomarker, tumor angiogenesis together, these findings indicate that MFAP2 has the potential to predict the prognosis of patients with HCC. MFAP2 also induces tumor cell proliferation and migration through EMT, and promotes tumor blood vessel formation through VEGFA, suggesting that MFAP2 may be a potential therapeutic target for HCC.

\section{Introduction}

Hepatocellular carcinoma (HCC) is the fifth most frequently diagnosed malignant cancer worldwide, with high mortality rates (1). China accounts for $\sim 55 \%$ of all global incidences of primary liver cancer, and has the third highest incidence rate of HCC worldwide, which has been attributed to high infection rates of hepatitis B virus (2). Radical tumor resection and liver transplantation are the most effective treatment options. However, owing to the difficulty of early detection, patients with HCC often lose the opportunity for surgical treatment due to local invasion and distant metastasis (3). Therefore, it is necessary to identify highly sensitive and specific diagnostic biomarkers to aid the early detection and prognostic prediction of patients with HCC.

Microfibril-associated protein 2 (MFAP2), also known as microfibril-associated glycoprotein (MAGP) 1 , interacts with microfibrillin to regulate microfibril functioning, and is the most widely distributed MAGP in most vertebrate proteins and microfiber components $(4,5)$. Previous studies have shown that the gene that codes MFAP2 is located at $1 \mathrm{p} 36.13$, and is associated with several malignant tumor types $(6,7)$. Consequently, MFAP2 is upregulated in breast cancer (8), thyroid cancer (9), melanoma (10), ovarian cancer (11) and gastric cancer $(12,13)$, and associated with the occurrence and progression of these tumors. Previous studies have also shown that MFAP2 is upregulated in HCC (14), but its role in the occurrence and development of the disease is unclear. In order to investigate the role of MFAP2 in the occurrence and development of $\mathrm{HCC}$, the aim of the present study was to evaluate the expression of MFAP2 in liver cancer by analyzing the tumor specimens and detailed clinical data of 94 patients. Based on the results from these clinical data, the mechanism underlying the effects of MFAP2 in HCC was also investigated. 


\section{Materials and methods}

Datasets and prediction. Prediction of MFAP2 expression was performed in HCC cases using datasets from The Cancer Genome Atlas (TCGA; https://portal.gdc.cancer. gov/projects/TCGA-LIHC). The TCGA dataset (LICH), downloaded for differential gene expression analysis in HCC, comprised 50 and 369 normal individuals and patients with HCC, respectively).

Patient enrollment and follow-up. Paraffin-embedded samples were obtained from total 94 patients with HCC, who underwent tumor resection at the General Surgery Department of the Anhui Provincial Hospital (Hefei, China) between 2010 and 2019, were enrolled in the current study. All patients were neither exposed to chemotherapy nor radiotherapy for anticancer treatment before surgery. Clinical information, including age, sex, tumor size and tumor node metastasis (TNM) status were recorded. Patients were most often followed up for 3 years (range, 0.1-3 years). The present study also included the fresh tissues of 21 patients who underwent tumor resection at the hospital between January 2019 and January 2020. The present study was performed in accordance with the Declaration of Helsinki, and was approved by the Ethics Committee of Anhui Medical University. The use of patient tissue samples and clinical information was approved by the patient, and written informed consent was obtained.

Immunohistochemistry. Tissues were fixed in $10 \%$ formalin for $9 \mathrm{~h}$ at room temperature, embedded in paraffin, and cut into $4-\mu \mathrm{m}$ thick sections using a microtome. The sections were heated in a $60^{\circ} \mathrm{C}$ oven for $2 \mathrm{~h}$ to soften the paraffin, and immediately transferred to xylene for dewaxing. The samples were rehydrated in descending concentrations of ethanol $(100$, 95 and 50\%) and then washed with PBS. Antigen retrieval was performed using sodium citrate solution in a microwave oven, after which the sections were cooled, and then exposed to $3 \%$ hydrogen peroxide to quench endogenous peroxidase activity. The sections were washed three times with PBS, and then incubated overnight with primary antibodies (Table I) in a humid chamber at $4^{\circ} \mathrm{C}$. Thereafter, slides containing tumor sections were washed 3 times with PBS (for $5 \mathrm{~min}$ each), and incubated with secondary antibody (Table I) at $37^{\circ} \mathrm{C}$ for $10 \mathrm{~min}$. Finally, the sections were incubated with 3-3 diaminobenzidine (Beijing Solarbio Science \& Technology Co., Ltd.), and the nuclei counterstained with hematoxylin. Then the samples were dehydrated with a gradient of alcohol $(50,95$ and $100 \%$ ) at room temperature, cleared with xylene, sealed with neutral gum, dried and observed using a light microscope (Nikon Corporation).

Immunohistochemical results were interpreted using a semi-quantitative evaluation system $(15,16)$. Briefly, staining intensity was scored as $0=$ absent, $1=$ weak, $2=$ medium, and $3=$ strong, while the percentage of the stained area was scored as $<10=1,10-50=2$, and $>50 \%=3$. A total score of $<3$ was considered low expression, and $\geq 3$ was regarded as high expression. All sections were scored in 5 fields under a high-power lens field, and an average score calculated. All sections were independently evaluated by two pathologists.
Western blot analysis. RIPA cell/tissue lysis buffer (Beyotime Institute of Biotechnology), containing a protease inhibitor mixture, was used to lyse the HCC and adjacent tissues. The contents were centrifuged at $13,000 \mathrm{x}$ g for $15 \mathrm{~min}$, and the protein-rich supernatant was collected. Protein concentration was determined using the BCA method (Beyotime Institute of Biotechnology); $40 \mu \mathrm{g} /$ lane protein was separated by $10 \%$ SDS-PAGE, and then transferred to a polyvinylidene fluoride membrane using the wet transfer method. The membranes were blocked with 5\% skimmed milk antigen, at room temperature for $1 \mathrm{~h}$, and then incubated overnight with primary antibodies (Table I) at $4^{\circ} \mathrm{C}$. The membranes were washed 3 times with Tris buffered saline/Tween $(0.1 \%)$ and then incubated at room temperature with secondary antibody (Table I) for $1.5 \mathrm{~h}$. Finally, an enhanced chemiluminescent reagent (Beyotime Institute of Biotechnology) was used to visualize the protein bands, which were quantified using Image Lab v3.1 software (Bio-Rad Laboratories, Inc.).

Reverse transcription-quantitative ( $R T-q) P C R$. Total RNA was isolated from HCC tissues and MHCC $97 \mathrm{H}$ cells using the RNAeasy ${ }^{\mathrm{TM}}$ Plus kit (Beyotime Institute of Biotechnology). A total of $1 \mu \mathrm{g}$ RNA was then converted to complementary DNA using the SuperScript IV reverse transcription kit (Thermo Fisher Scientific, Inc.) according to manufacturer's instructions. Levels of MFAP2 expression were determined via qPCR using the SYBR-Green ProFlex PCR system (Thermo Fisher Scientific, Inc.); the following thermocycling conditions were used: Initial denaturation at $95^{\circ} \mathrm{C}$ for $5 \mathrm{~min}$, followed by 40 cycles of $95^{\circ} \mathrm{C}$ for $30 \mathrm{sec}, 60^{\circ} \mathrm{C}$ for $30 \mathrm{sec}$ and $72^{\circ} \mathrm{C}$ for $30 \mathrm{sec}$. The corresponding primers are presented in Table II. Relative gene expression was determined using the $2^{-\Delta \Delta \mathrm{Cq}}$ method (17), relative to that of GAPDH.

Cell lines and transfection. The human HCC MHCC97H cell line and human umbilical vein endothelial cells (HUVECs) were acquired from the General Surgery Laboratory of Anhui Provincial Hospital. The MHCC97H cell line was cultured using RPMI-1640 medium containing 10\%, fetal bovine serum (FBS) and penicillin/streptomycin (100 U/ml) (all Gibco; Thermo Fisher Scientific, Inc.). HUVECs were cultured using endothelial cell medium (ECM; ScienCell Research Laboratories, Inc.). Both cell lines were maintained at $37^{\circ} \mathrm{C}$ with $5 \% \mathrm{CO}_{2}$ in a humidified incubator.

According to the manufacturer's protocol, on reaching $60 \%$ confluency, Lipofectamine ${ }^{\circledR} 2000$ (Gibco; Thermo Fisher Scientific, Inc.) was used to transfect the cells with MFAP2 small interfering RNA (siMFAP2 and MFAP2-siRNA) and negative control (NC)-siRNA (150 pmol/well), Shanghai Biosun Sci\&Tech Co; Table II). The cells were then cultured at $37^{\circ} \mathrm{C}$ in a low-serum medium (Opti-MEM; Gibco; Thermo Fisher Scientific, Inc.) for $6 \mathrm{~h}$. The medium was then replaced with complete medium containing $10 \%$ FBS, and the cells were incubated for a further $6 \mathrm{~h}$ prior to subsequent experimentation.

Cell counting kit $8(C C K-8)$ assay. The CCK-8 kit (Dojindo Molecular Technologies, Inc.) was used to assess the proliferative capacity of MHCC97H cells following MFAP2-knockdown. A 96-well plate was seeded with $3 \times 10^{3}$ cells/well, and $10 \mu \mathrm{l}$ CCK-8 reagent was added at 24, 48 and $72 \mathrm{~h}$. After incubating 
Table I. Antibodies used for western blotting and immunohistochemistry.

Dilution

\begin{tabular}{llccc} 
Antibody & \multicolumn{1}{c}{ Supplier } & Cat. no. & Western blotting & Immunohistochemistry \\
\cline { 4 - 5 } Rabbit anti-MFAP2 & ProteinTech Group, Inc. & AG24496 & $1: 1,000$ & $1: 200$ \\
Rabbit anti-E-cadherin & ProteinTech Group, Inc. & $20874-1-A P$ & $1: 1,000$ & $1: 300$ \\
Rabbit anti-N-cadherin & ProteinTech Group, Inc. & $22018-1-A P$ & $1: 1,000$ & $1: 100$ \\
Rabbit anti-vimentin & ProteinTech Group, Inc. & $10366-1-A P$ & $1: 1,000$ & $1: 200$ \\
Rabbit anti-VEGFA & ProteinTech Group, Inc. & $66828-1-$ Ig & $1: 1,000$ & - \\
Mouse anti-rabbit IgG & OriGene Technologies, Inc. & ZB-2301 & $1: 5,000$ & - \\
\hline
\end{tabular}

Table II. Quantitative PCR primer and siRNA sequences.

\begin{tabular}{ll}
\hline Primer/siRNA & \multicolumn{1}{c}{ Sequence } \\
\hline MFAP2 & Forward: 5'-TCCGCCGTGTGTACGTC \\
& ATT-3' \\
& Reverse: 5'-CTGGCCATCACGCCACA \\
& TTT-3' \\
GAPDH & Forward: 5'-TTGGTATCGTGGAAGGAC \\
& TCA-3' \\
& Reverse: 5'-TGTCATCATATTTGGCAG \\
& GTT-3' \\
MFAP2-siRNA & Sense: 5'-CCCACUAUAGCGACCAGA \\
& UTT-3' \\
& Antisense: 5'-AUCUGGUCGCUAUAG \\
& UGGGTT-3' \\
& Sense: 5'-CCAUACACAGGCCUUGCA \\
NC-siRNA & ATT-3' \\
& Antisense: 5'-UUGCAAGGCCUGUGU \\
& AUGGTT-3'
\end{tabular}

MFAP2, microfibrillar-associated protein 2; NC, negative control; si, small interfering (RNA).

for a further hour, the OD value of each well was measured at $450 \mathrm{~nm}$.

Transwell assay. A total of $1 \times 10^{5} \mathrm{MHCC} 97 \mathrm{H}$ cells were seeded into the upper chamber of a Transwell insert (pore size, $8-\mu \mathrm{m}$ ) containing Matrigel (Costar; Corning, Inc.), with $200 \mu \mathrm{l}$ serum-free medium; $500 \mu \mathrm{l}$ complete medium was added to the lower chamber, and the cells were cultured at $37^{\circ} \mathrm{C}$ for $24 \mathrm{~h}$. The migratory cells were fixed with $4 \%$ formaldehyde at room temperature for $20 \mathrm{~min}$, and then stained with $0.2 \%$ crystal violet at room temperature for $20 \mathrm{~min}$. The cells were counted in five random microscopic fields using a light microscope (magnification, $\mathrm{x} 200$ ).

Wound-healing assay. A total of $3 \times 10^{5} \mathrm{MHCC} 97 \mathrm{H}$ cells per well were seeded into a 6-well plate and cultured for $24 \mathrm{~h}$ at $37^{\circ} \mathrm{C}$. The cells were then cultured in serum-free basal medium for $6 \mathrm{~h}$, and a $1,000-\mu 1$ pipette tip was used to mark out the cell-free area, prior to continued culture for an additional $24 \mathrm{~h}$. Images were captured under a light microscope (Nikon Corporation; magnification, x100). The area of the cell-free zone was recorded, and the wound area was determined using the following formula: (Initial wound area-final wound area)/initial wound area x $100 \%$.

Tube-formation experiment. Pre-chilled Matrigel (100 $\mu \mathrm{l} /$ well) was added to a 24-well plate and incubated at $37^{\circ} \mathrm{C}$ for $1 \mathrm{~h}$. Then, $1 \times 10^{4}$ HUVECs were seeded into each well with $200 \mu \mathrm{l}$ ECM medium, as well as $200 \mu \mathrm{l}$ supernatant from MHCC97H cells cultured in serum-free medium (obtained by centrifugation at $12,000 \mathrm{x} \mathrm{g}$ for $3 \mathrm{~min}$ at $4^{\circ} \mathrm{C}$ ). After $7 \mathrm{~h}$, images were captured using a light microscope (Nikon Corporation; magnification, $\mathrm{x} 100$ ), and analyzed using Image J software (V.1.8.0; National Institutes of health).

Statistical analysis. The $\chi^{2}$ test was used to compare immunohistochemistry scores, and $\chi^{2}$ test or Fisher's exact test were used to determine the association between clinicopathological characteristics and MFAP2 expression. One-way analysis of variance and Tukey's post-hoc test were used for multiple comparisons of means, and a two-tailed unpaired Student's t test was used to compare the differences between two groups. For data conforming to normal distribution and homogeneity of variance, the differences between two paired groups (normal vs. tumor tissues) were assessed using paired Student's t-test. Spearman's rank correlation was used to evaluate the association between the expression profiles of MFAP2 and epithelial-mesenchymal transition (EMT)-related proteins. Kaplan-Meier survival curves were used to analyze the disease-free survival (DFS) and overall survival (OS) of patients with HCC; differences were assessed using the log-rank test. Cox proportional hazard regression models were used to obtain unadjusted and the adjusted hazard ratio (HR) and 95\% confidence interval (CI) values for survival in univariate and multivariate analysis. All statistical analyses were performed using SPSS 22 (IBM Corp) and GraphPad Prism 14 (GraphPad Software, Inc.). Data are expressed as the mean $\pm \mathrm{SD}$, and $\mathrm{P}<0.05$ was considered to indicate a statistically significant difference.

\section{Results}

MFAP2 is upregulated in HCC. Immunohistochemistry results revealed that MFAP2 was significantly upregulated 
A

$$
\text { MFAP2 }
$$

Tumor

Control

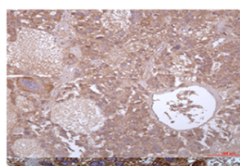

$$
\text { Vimentin }
$$

$$
\mathrm{N} \text {-cadherin }
$$

E-cadherin
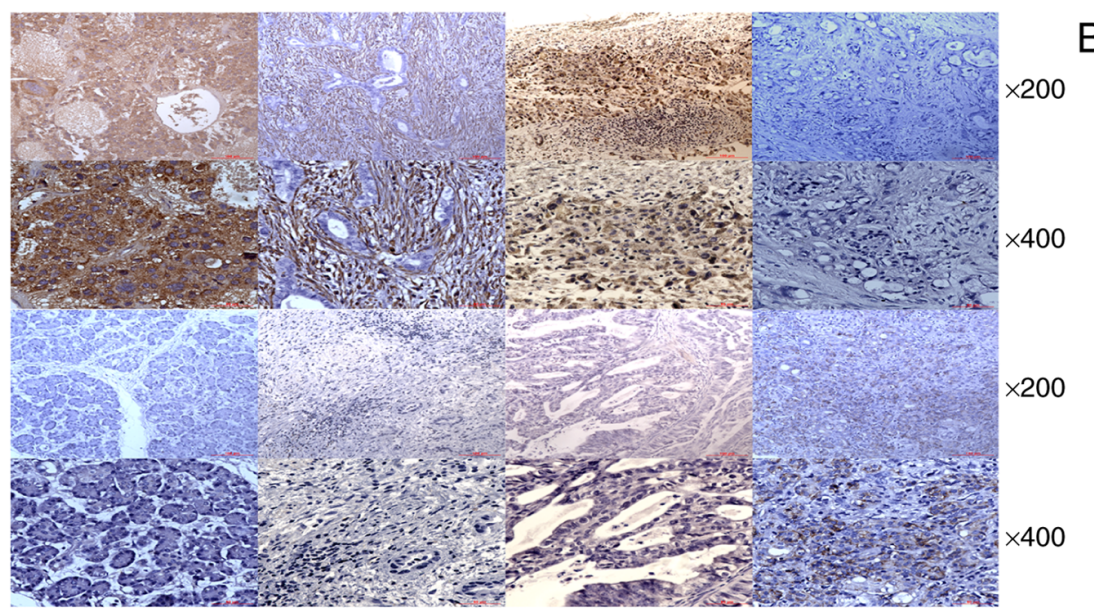

B
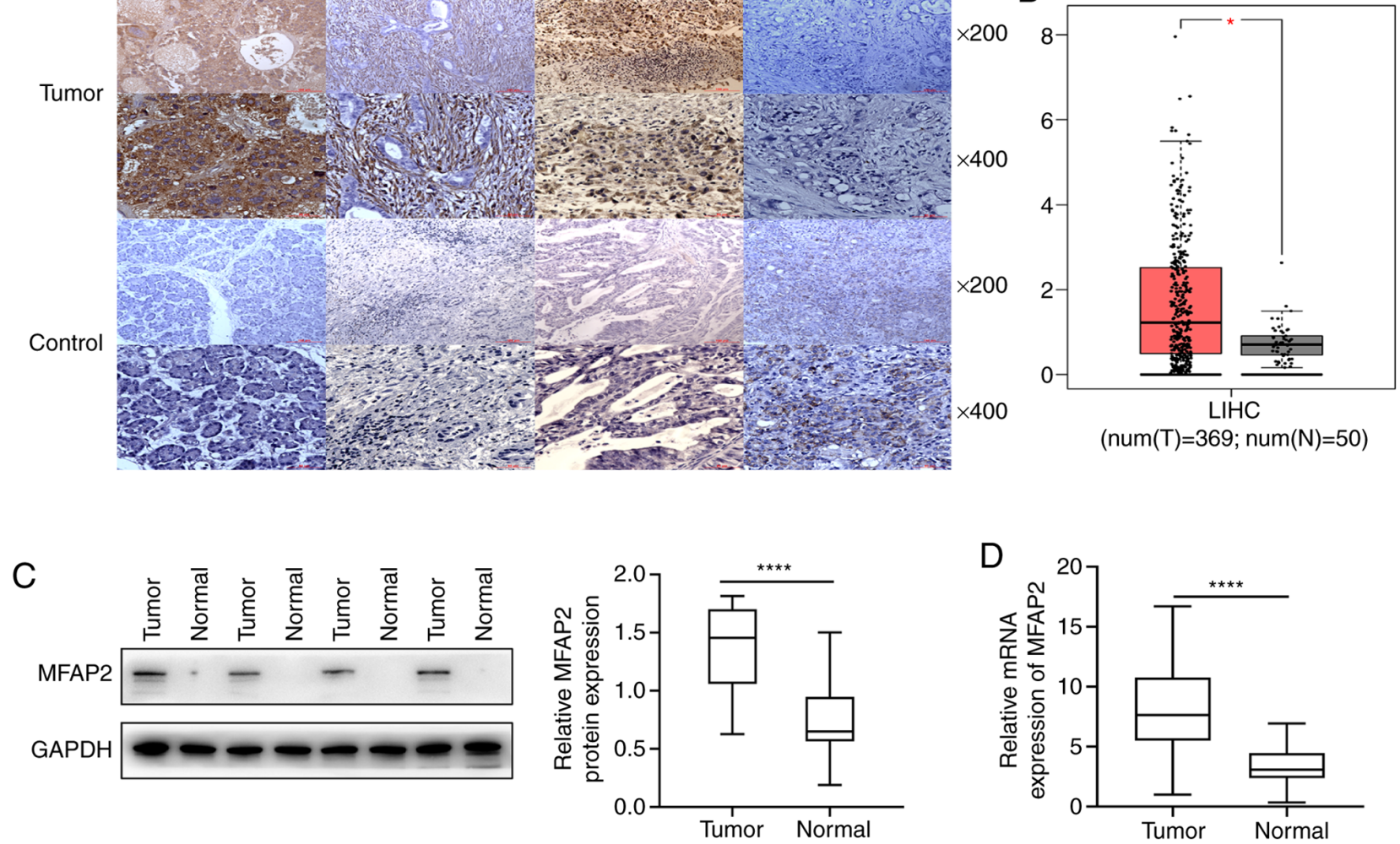

D

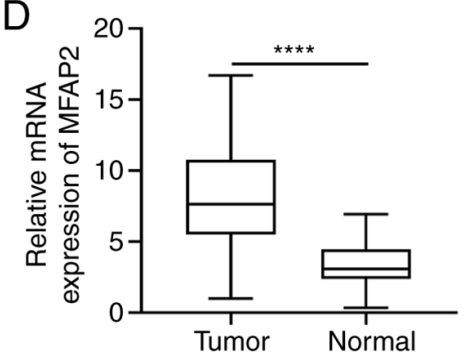

Figure 1. Expression of MFAP2 and epithelial-mesenchymal transition-related proteins in HCC. (A) Immunohistochemical staining for MFAP2, vimentin, N-cadherin and E-cadherin. Magnification, x200 and x400. (B) MFAP2 expression in HCC tissues and paired normal tissues (TCGA-LIHC). (C) Representative images of MFAP2 protein expression in HCC tissues and paired normal tissues from four patients. Western blot analysis of MFAP2 expression in 21 pairs of HCC tissues and matched adjacent normal tissues. (D) Reverse transcription-quantitative PCR analysis of MFAP2 expression in 21 pairs of HCC tissues and matched adjacent normal tissues. ${ }^{*} \mathrm{P}<0.05$ and ${ }^{* * * *} \mathrm{P}<0.0001$. MFAP2, microfibrillar-associated protein 2; HCC, hepatocellular carcinoma; LIHC, liver hepatocellular carcinoma; T, tumor; N, normal.

in $76.6 \%$ of HCC tissues (72 of 94; Fig. 1A and Table III). The expression of MFAP2 in HCC tissues from TCGA was also higher than that in normal tissues $(\mathrm{P}<0.05$; Fig. $1 \mathrm{~B})$. A comparison of patterns of MFAP2 expression in 21 pairs of fresh carcinoma and liver tissues revealed a significant upregulation in liver cancer, relative to adjacent normal tissues $(\mathrm{P}<0.001$; Fig. 1C). RT-qPCR corroborated the western blots findings, as evidenced by a significant upregulation in MFAP2 mRNA expression in fresh HCC compared with normal tissues $(\mathrm{P}<0.001$; Fig. 1D). These results indicate that MFAP2 is upregulated in HCC.

MFAP2 expression is positively correlated with that of EMT marker proteins. Immunohistochemistry results revealed high $\mathrm{N}$-cadherin protein expression in $74.5 \%$ (70 of 94) in HCC specimens (Fig. 1 and Table IV). Spearman's correlation analysis revealed a significant positive correlation between MFAP2 expression and that of $\mathrm{N}$-cadherin $(\mathrm{r}=0.425, \mathrm{P}=0.021$; Table IV). High vimentin expression was also detected in HCC tissues (77/94, 81.9\%; Fig. 1), which was also positively correlated with MFAP2 ( $\mathrm{r}=0.393$, $\mathrm{P}=0.037$; Table IV). By contrast, E-cadherin, an epithelial phenotype-related protein, was downregulated in $\mathrm{HCC}$ tissues (Fig. 1A), and further showed a significant negative correlation with MFAP2 expression ( $\mathrm{r}=-0.204, \mathrm{P}=0.049$; Table IV). These results suggest that the expression of
Table III. Differential expression of MFAP2 in HCC tissues and corresponding paracarcinoma tissues $(n=94)$.

\begin{tabular}{llll}
\hline & \multicolumn{2}{c}{ MFAP2 expression } & \\
\cline { 2 - 3 } Tissue & Low, n (\%) & High, n (\%) & P-value \\
\hline HCC & $22(23.4)$ & $72(76.6)$ & $<0.05$ \\
Paracarcinoma & $51(54.3)$ & $43(45.7)$ & \\
\hline
\end{tabular}

HCC, hepatocellular carcinoma; MFAP2, microfibrillar-associated protein 2 .

MFAP2 is positively correlated with the expression of EMT marker proteins.

Association between MFAP2 expression and patient clinical features. Significant associations between MFAP2 expression and clinical characteristics were assessed across the 94 patients with HCC analyzed (Table V). There was no correlation between MFAP2 expression and age, sex, tumor size, tumor nodule number, tumor capsula, liver cirrhosis, $\mathrm{HBeAg}$ status and Child-Pugh grade. However, MFAP2 expression was positively correlated with other clinical parameters, such as vascular invasion $(\mathrm{P}=0.016)$, Edmondson grade $(\mathrm{P}=0.006)$, 

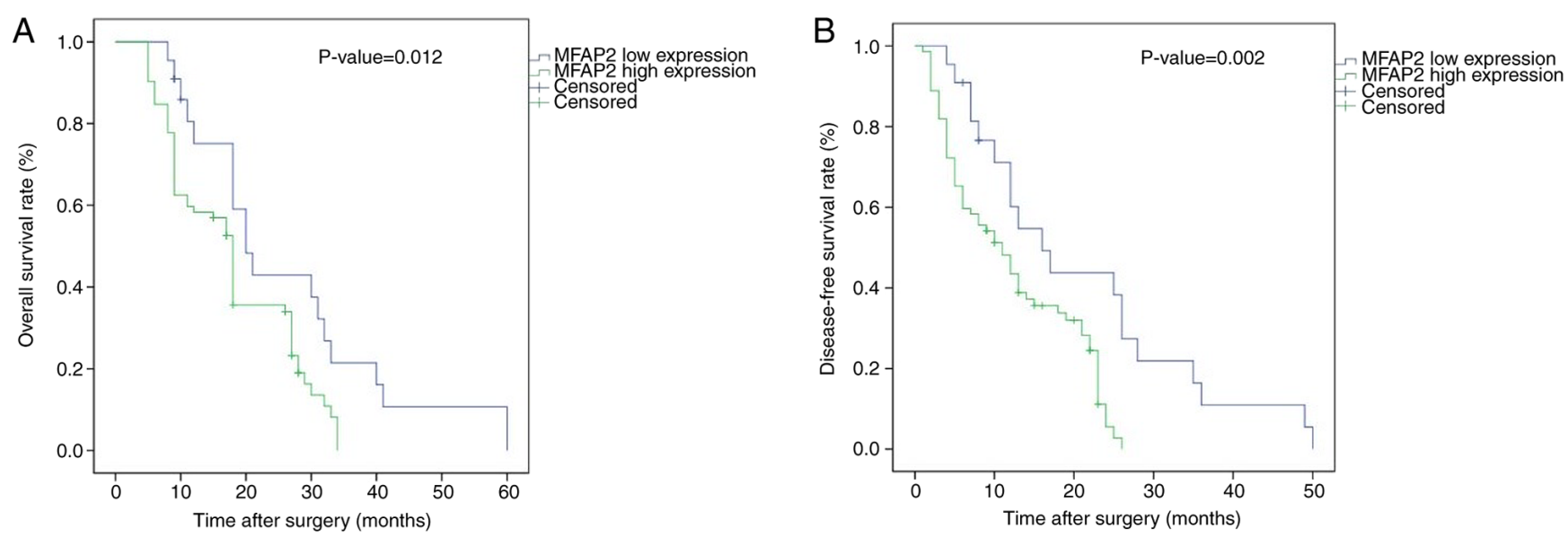

Figure 2. Kaplan-Meier analysis of (A) overall and (B) disease-free survival in patients with hepatocellular carcinoma according to MFAP2 staining. MFAP2, microfibrillar-associated protein 2.

Table IV. Association between MFAP2 expression and that of E-cadherin, N-cadherin and vimentin in hepatocellular carcinoma.

\begin{tabular}{lrrrr}
\hline & \multicolumn{2}{c}{$\begin{array}{c}\text { MFAP2 } \\
\text { expression, } n\end{array}$} & & \\
\cline { 2 - 3 } Immunoreactivity & Low & High & r-value & P-value \\
\hline E-cadherin expression & & & & \\
$\quad$ Low & 14 & 60 & -0.204 & 0.049 \\
High & 8 & 12 & & \\
N-cadherin expression & & & & \\
Low & 13 & 11 & 0.425 & 0.021 \\
$\quad$ High & 9 & 61 & & \\
Vimentin expression & & & & \\
$\quad$ Low & 10 & 7 & 0.393 & 0.037 \\
High & 12 & 65 & & \\
\hline
\end{tabular}

MFAP2, microfibrillar-associated protein 2.

TNM status $(\mathrm{P}=0.027)$ and AFP level $(\mathrm{P}=0.0006)$. These data indicate that the expression of MFAP2 is associated with a number of clinical features, and subsequently, with poor patient prognosis.

Survival analyses. Kaplan-Meier curves were generated, and the log-rank test revealed that patients with high MFAP2 expression exhibited shorter DFS and OS times than those with low MFAP2 expression (Fig. 2). The median DFS and OS for patients with high MFAP2 expression were 10 and 17 months, respectively, whereas those for patients with low MFAP2 expression were 12.5 (DFS) and 19 (OS) months. Results from univariate analysis showed that high MFAP2 expression, high vascular invasion, Edmundson grade, TNM stage and AFP level were predictors for poor OS and DFS in patients with HCC (Table VI). On the other hand, multivariate survival analysis revealed that MFAP2 expression was an independent predictor for DFS $(\mathrm{HR}=3.323 ; 95 \% \mathrm{CI}=1.652-6.216 ; \mathrm{P}<0.001)$ and $\mathrm{OS}(\mathrm{HR}=3.895 ; 95 \% \mathrm{CI}=1.955-7.029 ; \mathrm{P}<0.001)$.

MFAP2-knockdown inhibits the proliferation and invasiveness of MHCC $97 \mathrm{H}$ cells. The effect of MFAP2 on the progression of HCC was further evaluated using in vitro experiments. siRNA was used to knock down MFAP2 in MHCC97H cells (Fig. 3A and B), after which cellular proliferation and migration were evaluated. Compared with the NC group, the CCK- 8 assay results showed that knocking down MFAP2 significantly reduced the proliferation of $\mathrm{MHCC} 97 \mathrm{H}$ cells (Fig. 3C). Transwell and wound-healing experiments revealed that following MFAP2-knockdown, the migration and invasion ability of MHCC97H cells was also reduced (Fig. 3D and E). These data indicate that MFAP2-knockdown inhibited the proliferation and invasiveness of MHCC97H cells.

Effects of MFAP2-knockdown on EMT. As shown in Table IV, the expression of MFAP2 in human HCC tissues was correlated with EMT-related protein expression. Since EMT is a key event that affects tumor differentiation and metastasis, the effect of MFAP2 on EMT was further investigated by detecting EMT-specific proteins (including E-cadherin, $\mathrm{N}$-cadherin and vimentin). After silencing the expression of MFAP2 in MHCC97H cells, compared with the NC and the Mock group, western blot analyses showed that the expression of E-cadherin increased, while the expression of N-cadherin and vimentin decreased, indicating that MFAP2 is essential for the EMT of MHCC97H cells (Fig. 4A and B).

Effects of MFAP2 on tube formation. Vascular endothelial growth factor A (VEGFA) is the most important VEGF in tumors (18). In the present study, the expression of VEGFA was significantly reduced following MFAP2-knockdown (Fig. 4B). Next, an in vitro HUVEC tube-formation assay was used to directly determine the effect of MFAP2 on angiogenesis. Compared with the NC group, the supernatant of MFAP2-knockout MHCC97H cells reduced the average tube length, network and branch point number of HUVECs (Fig. 4C). These findings indicated that silencing MFAP2 reduces blood vessel formation. 
Table V. MFAP2 expression status in relation to clinicopathologic features in 94 patients with hepatocellular carcinoma.

\begin{tabular}{|c|c|c|c|c|c|}
\hline \multirow[b]{2}{*}{ Parameter } & \multirow[b]{2}{*}{ Patients, $\mathrm{n}$} & \multicolumn{2}{|c|}{ MFAP2 expression, $\mathrm{n}$} & \multirow[b]{2}{*}{$\chi^{2}$-value } & \multirow[b]{2}{*}{ P-value } \\
\hline & & Low & High & & \\
\hline \multicolumn{6}{|l|}{ Age } \\
\hline$\leq 60$ & 69 & 14 & 55 & 1.404 & 0.236 \\
\hline$>60$ & 25 & 8 & 17 & & \\
\hline \multicolumn{6}{|l|}{ Sex } \\
\hline Male & 59 & 11 & 48 & 2.003 & 0.157 \\
\hline Female & 35 & 11 & 24 & & \\
\hline \multicolumn{6}{|c|}{ Tumor size, cm } \\
\hline$\leq 5$ & 32 & 10 & 22 & 1.666 & 0.197 \\
\hline$>5$ & 62 & 12 & 50 & & \\
\hline \multicolumn{6}{|c|}{ Tumor nodule number } \\
\hline Single & 76 & 17 & 59 & 0.238 & 0.626 \\
\hline Multiple & 18 & 5 & 13 & & \\
\hline \multicolumn{6}{|c|}{ Vascular invasion } \\
\hline Yes & 33 & 3 & 30 & 5.812 & 0.016 \\
\hline No & 61 & 19 & 42 & & \\
\hline \multicolumn{6}{|c|}{ Tumor capsula } \\
\hline Absent & 38 & 10 & 28 & 0.302 & 0.583 \\
\hline Present & 56 & 12 & 44 & & \\
\hline \multicolumn{6}{|c|}{ Edmondson grade } \\
\hline $\mathrm{I}-\mathrm{II}$ & 53 & 18 & 35 & 7.556 & 0.006 \\
\hline III-IV & 41 & 4 & 37 & & \\
\hline \multicolumn{6}{|l|}{ Cirrhosis } \\
\hline Absent & 31 & 9 & 22 & 0.817 & 0.366 \\
\hline Present & 63 & 13 & 50 & & \\
\hline \multicolumn{6}{|c|}{ HBeAg status } \\
\hline Positive & 68 & 14 & 54 & 1.088 & 0.297 \\
\hline Negative & 26 & 8 & 18 & & \\
\hline \multicolumn{6}{|c|}{ Child-Pugh grade } \\
\hline A & 86 & 20 & 66 & 0.12 & 1.000 \\
\hline B & 8 & 2 & 6 & & \\
\hline \multicolumn{6}{|l|}{ TNM stage } \\
\hline I-II & 58 & 18 & 40 & 4.918 & 0.027 \\
\hline III-IV & 36 & 4 & 32 & & \\
\hline \multicolumn{6}{|c|}{$\operatorname{AFP}(\mathrm{ng} / \mathrm{ml})$} \\
\hline$\leq 20$ & 20 & 14 & 6 & 27.56 & 0.0006 \\
\hline$>20$ & 74 & 8 & 66 & & \\
\hline
\end{tabular}

MFAP2, microfibrillar-associated protein 2.

\section{Discussion}

MFAP2, a component of microfibers which was originally identified in ligaments (19), interacts with numerous elastic fiber components, such as tropoelastin and decorin, hence is considered a component of elastin (20). Previous studies have shown that an interaction between MFAP2 and fibrillin 1 can regulate the number of osteoclasts and bone resorption (21). In addition, MFAP2 has been shown to play an important role in tissue homeostasis and differentiation (5-7). However, disease-induced fibrillin mutations may alter the ability of myofibrils to bind MFAP2, and lead to the occurrence and deterioration of the disease. Although some reports have described MFAP2 upregulation in HCC (14), its exact role therein remains unclear. 
Table VI. Univariate and multivariate analysis of factors associated with overall and Disease-free survival.

A, Univariate analysis

\begin{tabular}{|c|c|c|c|c|c|c|}
\hline \multirow[b]{2}{*}{ Parameter } & \multicolumn{3}{|c|}{ Overall survival } & \multicolumn{3}{|c|}{ Disease-free survival } \\
\hline & HR & $95 \% \mathrm{CI}$ & P-value & HR & $95 \% \mathrm{CI}$ & P-value \\
\hline Age, $\leq 60$ vs. $>60$ years & 0.923 & $0.552-1.469$ & 0.796 & 0.935 & $0.556-1.478$ & 0.789 \\
\hline Sex, male vs. female & 0.956 & $0.587-1.523$ & 0.778 & 0.964 & $0.590-1.511$ & 0.812 \\
\hline Tumor size, $\leq 5$ vs. $>5 \mathrm{~cm}$ & 0.962 & $0.481-1.874$ & 0.912 & 0.945 & $0.519-1.727$ & 0.853 \\
\hline Tumor nodules, single vs. multiple & 1.326 & $0.831-2.127$ & 0.246 & 1.285 & $0.827-2.012$ & 0.266 \\
\hline Vascular invasion, yes vs. no & 0.635 & $0.236-0.843$ & 0.016 & 0.543 & $0.313-0.914$ & 0.014 \\
\hline Tumor capsula, absent vs. present & 0.594 & $0.329-1.026$ & 0.068 & 0.654 & $0.367-1.026$ & 0.091 \\
\hline Edmondson grade, I-II vs. III-IV & 2.627 & $1.567-4.765$ & $<0.001$ & 2.357 & $1.428-4.458$ & 0.003 \\
\hline Cirrhosis, absent vs. present & 1.252 & $0.629-2.433$ & 0.484 & 1.117 & $0.541-2.110$ & 0.727 \\
\hline HBeAg status, positive vs. negative & 0.852 & $0.423-1.621$ & 0.652 & 0.721 & $0.323-1.435$ & 0.336 \\
\hline Child-Pugh grade, A vs. B & 1.061 & $0.623-1.811$ & 0.799 & 1.146 & $0.686-1.915$ & 0.603 \\
\hline TNM stage, I-II vs. III-IV & 2.222 & $1.375-3.589$ & 0.001 & 2.331 & $1.440-3.774$ & 0.001 \\
\hline AFP, $\leq 20 \mathrm{vs} .>20 \mathrm{U} / \mathrm{ml}$ & 1.934 & $1.074-3.483$ & 0.028 & 1.759 & $1.000-3.095$ & 0.052 \\
\hline MFAP2 expression, high vs. low & 2.003 & $1.150-3.488$ & 0.014 & 2.151 & $1.228-3.768$ & 0.007 \\
\hline
\end{tabular}

B, Multivariate analysis

\begin{tabular}{|c|c|c|c|c|c|c|}
\hline \multirow[b]{2}{*}{ Parameter } & \multicolumn{3}{|c|}{ Overall survival } & \multicolumn{3}{|c|}{ Disease-free survival } \\
\hline & HR & $95 \% \mathrm{CI}$ & P-value & HR & $95 \% \mathrm{CI}$ & P-value \\
\hline Vascular invasion, yes vs. no & 5.141 & $2.534-10.427$ & $<0.001$ & 12.626 & $5.007-31.840$ & $<0.001$ \\
\hline Edmondson grade, I-II vs. III-IV & 3.647 & $2.567-5.765$ & $<0.001$ & 3.324 & $2.428-5.458$ & 0.023 \\
\hline TNM stage, I-II vs. III-IV & 3.222 & $2.375-4.589$ & $<0.001$ & 3.351 & $2.440-8.774$ & $<0.001$ \\
\hline AFP,$\leq 20$ vs. $>20 \mathrm{U} / \mathrm{ml}$ & 2.932 & $1.324-3.453$ & 0.038 & 1.759 & $1.500-3.045$ & 0.022 \\
\hline MFAP2 expression, high vs. low & 3.895 & $1.955-7.029$ & $<0.001$ & 3.323 & $1.652-6.216$ & $<0.001$ \\
\hline
\end{tabular}

HR, hazard ratio; CI confidence interval; MFAP2, microfibrillar-associated protein 2.

The present study revealed that MFAP2 was upregulated in liver cancer, relative to normal adjacent tissues. These results were consistent with those from TCGA database. Furthermore, correlation analyses revealed a significant positive correlation between MFAP2 upregulation and vascular invasion, high Edmondson grade, elevated serum AFP and TNM stage in HCC tumors. In addition, results from Kaplan-Meier survival analysis indicated that patients with high MFAP2 expression showed poorer OS and DFS, relative to those with low expression, and Cox regression analysis also confirmed that MFAP2 upregulation was an independent predictor for poor OS and DFS in patients with HCC.

Through in vitro experimentation, silencing MFAP2 was found to significantly reduce the proliferation and invasion abilities of HCC cell lines, which indirectly indicates that MFAP2 affects the progression of HCC. Tumor progression is a complex process regulated by numerous genes over multiple steps, whereas EMT is a process of self-repair that occurs after damage to adult epidermal cells $(6,19)$. During tumor cell development, EMT causes the loss of epithelial characteristics and gain of mesenchymal cell traits $(22,23)$, bestowing tumor cells with stronger invasion and migration capabilities. Downregulation of E-cadherin, which is associated with cellular adhesion, is considered the most significant feature of EMT (24-26). At the same time, interstitial factors associated with tumor cells, such as Snail1, Snail2, Twist and MMP-2, are often upregulated $(27,28)$. In the present study, it was evident that MFAP2 knockdown was accompanied by the upregulation of vimentin and E-cadherin, while N-cadherin was downregulated as a result of MFAP2-knockdown. These results indicate that MFAP2 may regulate the invasion and metastasis of HCC via EMT.

In addition to EMT, angiogenesis also plays an important role tumor invasion and metastasis (29). There are two distinct phases of tumor growth, that is, from the avascular slow growth phase to the vascular fast proliferation phase. Tumor angiogenesis enables HCC and other solid tumors to obtain sufficient nutrients, which is a key link to promote the rapid growth of tumors (30).

Angiogenesis stimulating factors include a class of cytokines known as antigenic growth factors, which act to stimulate the formation of blood vessels (31). At present, 
A

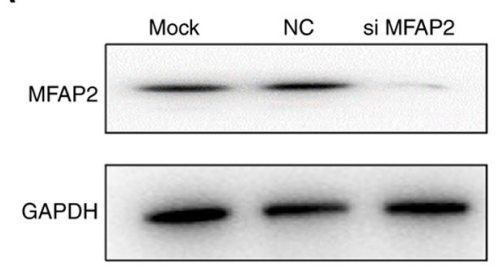

C
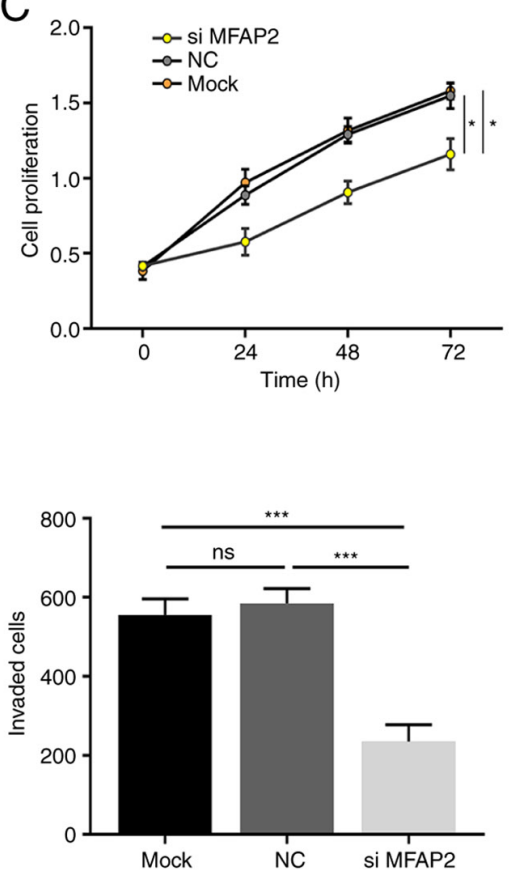
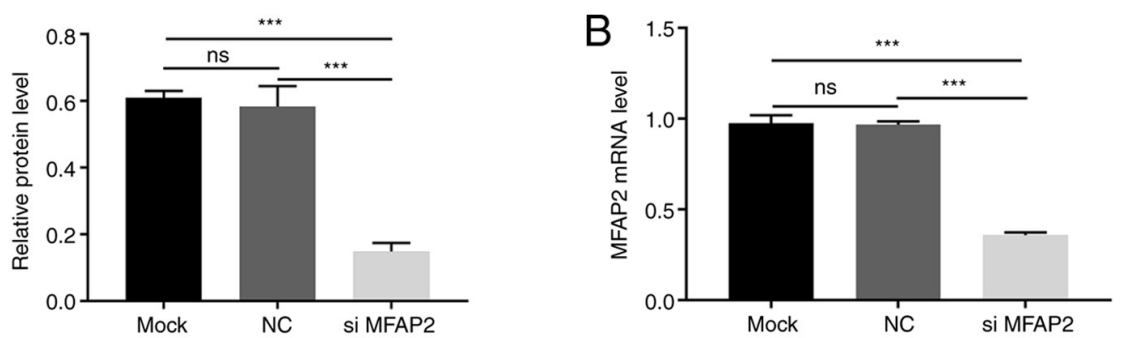

D

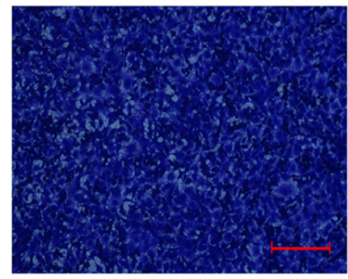

Mock

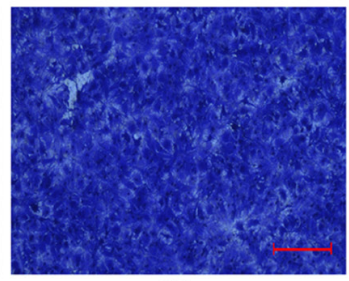

NC
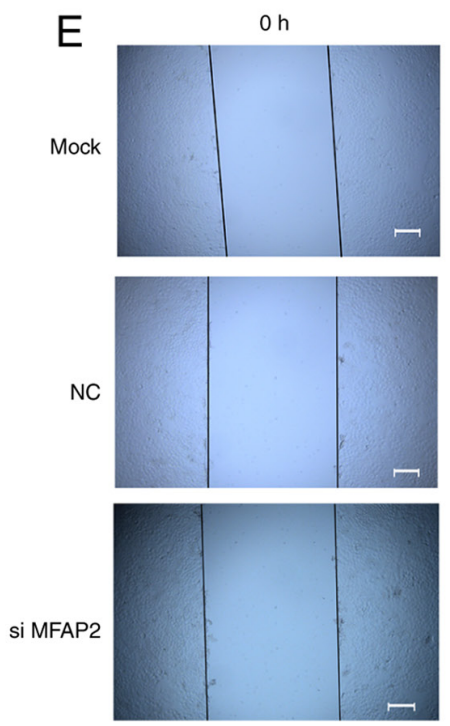
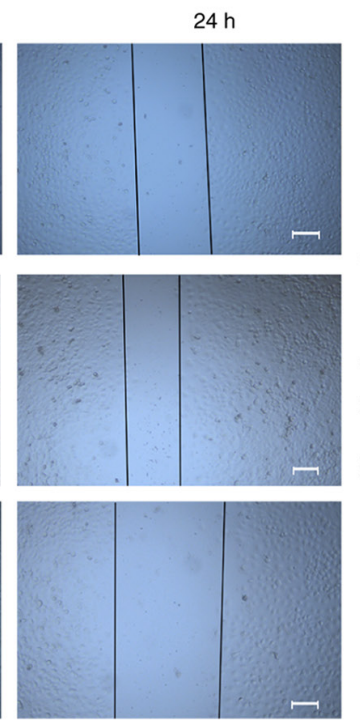
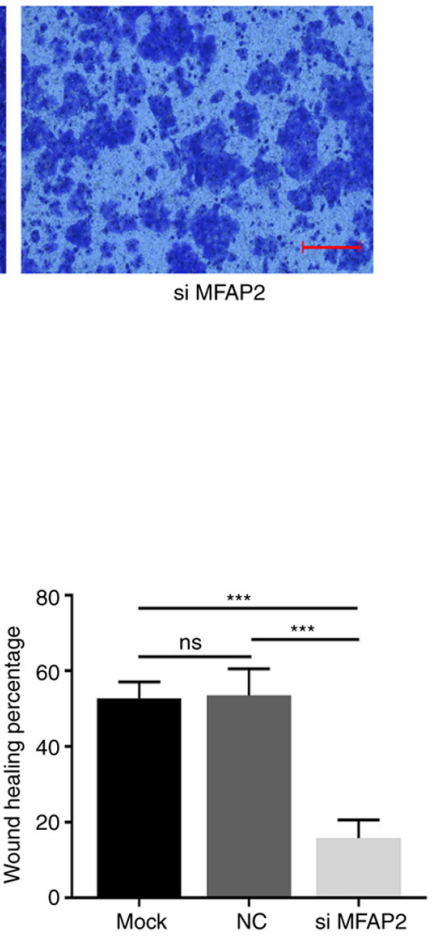

Figure 3. MFAP2-knockdown decreases the proliferation, migration and invasion capabilities of liver cancer cells. MFAP2-knockdown in MHCC97H cells was verified by (A) western blotting and (B) reverse transcription-quantitative PCR. (C) Proliferation of MHCC97H cells was detected by Cell Counting Kit 8 analysis after MFAP2-knockdown. (D) Migration of MHCC97H cells was assessed by Transwell assay. (E) Wound-healing assay was used to evaluate the migration ability of MHCC $97 \mathrm{H}$ cells. Data are presented as the mean \pm SD of three independent experiments. ${ }^{*} \mathrm{P}<0.05$ and ${ }^{* * * *} \mathrm{P}<0.001$. MFAP2, microfibrillar-associated protein 2; NC, negative control; si, small interfering (RNA).

angiogenic factors such as platelet-derived growth factor, fibroblast growth factor, transforming growth factor and VEGFA have been discovered (32), of which VEGFA plays a key role in tumor angiogenesis (33). When tumor cells develop, their expression levels will generally rise quickly. Studies have shown that VEGFA is associated with tumor invasiveness and susceptibility (34). Currently, systemic therapies, including molecularly targeted drugs and immune checkpoint inhibitors, have become common complementary therapies for patients with HCC. VEGF-targeted therapeutic drugs, including sorafenib and lenvatinib, are often used to inhibit the formation of blood vessels in liver cancer. In the present study, clinical data revealed that MFAP2 was associated with tumor angiogenesis, and in vitro experimentation indicated that MFAP2-knockdown was accompanied by low expression levels of VEGF. Therefore, we hypothesize that MFAP2 may affect the formation of tumor blood vessels by influencing VEGF. Inhibitors of MFAP2 may therefore be combined with VEGF inhibitors to retard the formation of tumor blood vessels.

In summary, the results of the present study suggest that MFAP2 upregulation promotes tumor progression, is associated with poor patient prognosis, and is an independent prognostic biomarker for poor prognosis in HCC. Through in vitro experimentation, MFAP2 was shown to inhibit the proliferation and migration of $\mathrm{MHCC} 97 \mathrm{H}$ cells by affecting the EMT process. In addition, MFAP2 reduced the formation of tumor blood vessels by inhibiting the expression of VEGFA. These results indicate that the use of certain specific inhibitors to block MFAP2 activity may provide 
A
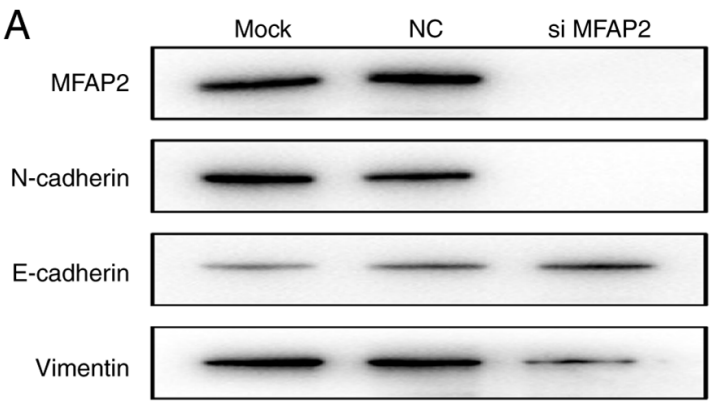
GAPDH
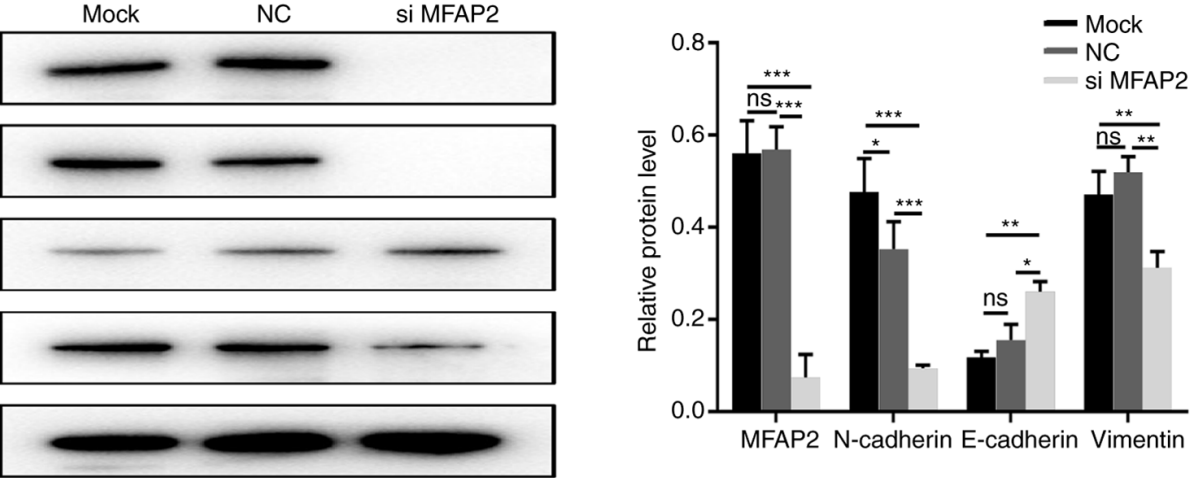

B
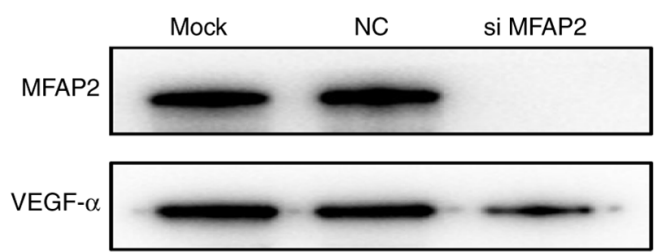
GAPDH
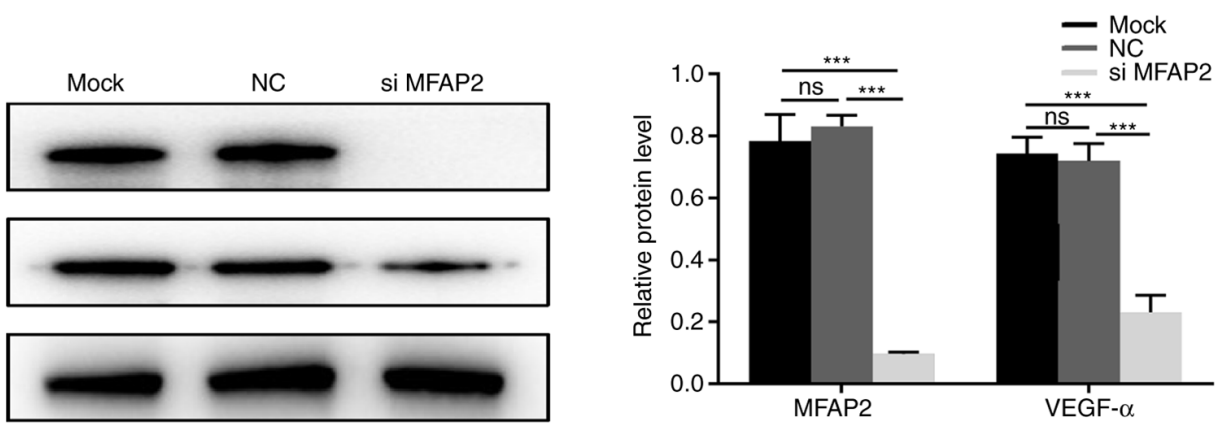

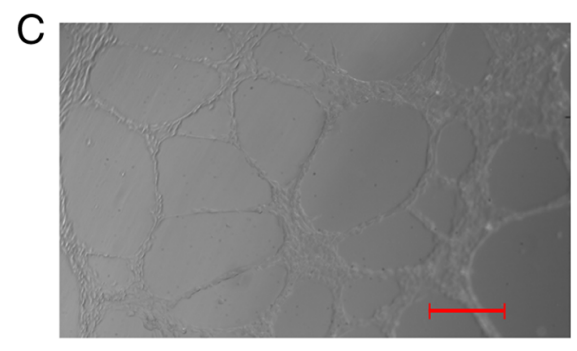

Mock

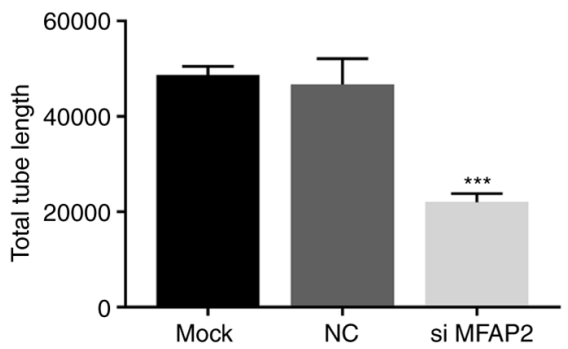

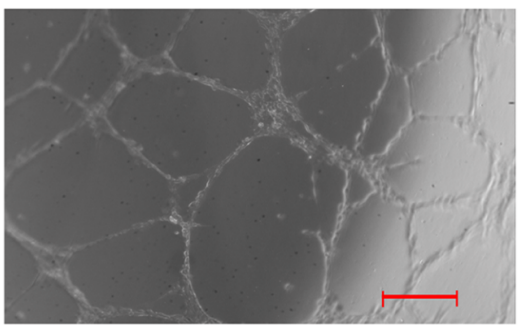

NC

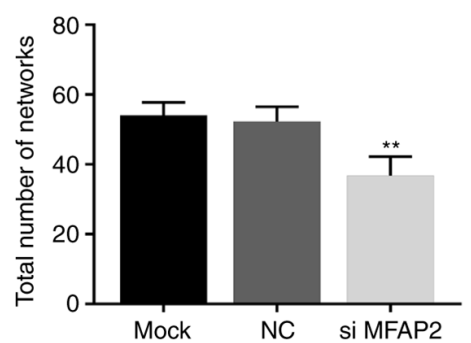

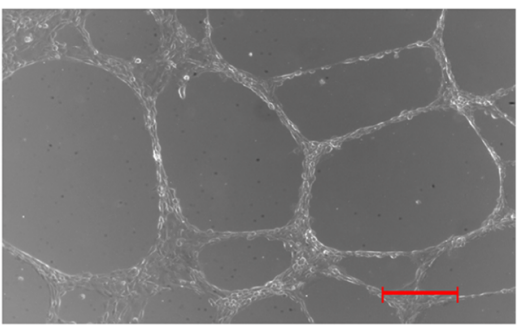

si MFAP2

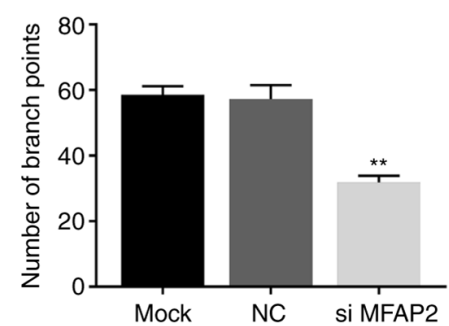

Figure 4. MFAP2-knockdown alters epithelial-mesenchymal transition and blood vessel formation. (A) Changes in the expression of epithelial-mesenchymal transition-associated proteins, N-cadherin, vimentin and E-cadherin, after MFAP2-knockdown were detected by western blotting. (B) Changes in the expression of VEGFA after MFAP2-knockdown were detected by western blot analysis. (C) Effects of MFAP2 on human umbilical vein endothelial cell tube formation. Total tube length, number of branch points and total number of networks in captured images were measured using ImageJ. Data are presented as the mean \pm SD of three independent experiments. siMFAP2 group vs. Mock and NC group ${ }^{*} \mathrm{P}<0.05,{ }^{* *} \mathrm{P}<0.01$ and ${ }^{* * * *} \mathrm{P}<0.001$. MFAP2, microfibrillar-associated protein 2; NC, negative control; si, small interfering (RNA); VEGFA, vascular endothelial cell growth factor A.

novel targets for the development of anti-angiogenic therapies against liver cancer.

\section{Acknowledgements}

Not applicable.

\section{Funding}

No funding was received.

\section{Availability of data and materials}

The datasets used and/or analyzed during the current study are available from the corresponding author on reasonable request.

\section{Authors' contributions}

$\mathrm{NZ}$ and WJ designed the study, and performed the experiments alongside FS. WJ wrote the manuscript and analyzed the data alongside FS. WJ and FS conceived the study, supervised 
the experiments and revised the manuscript for important intellectual content. All authors read and approved the final manuscript. ZN, FS and WJ confirm the authenticity of all the raw data.

\section{Ethics approval and consent to participate}

The present study was approved by the Ethics Committee of Anhui Medical University (approval no. 2019-SH-010). All patients provided written informed consent.

\section{Patient consent for publication}

Not applicable.

\section{Competing interests}

The authors declare that they have no competing interests.

\section{References}

1. Jemal A, Bray F, Center MM, Ferlay J, Ward E and Forman D Global cancer statistics. CA Cancer J Clin 61: 69-90, 2011.

2. Shen JD, Fu SZ, Ju LL, Wang YF, Dai F, Liu ZX, Ji HZ, Shao JG and Bian ZL: High expression of ubiquitin-conjugating enzyme E2A predicts poor prognosis in hepatocellular carcinoma. Oncol Lett 15: 7362-7368, 2018

3. Zhai X, Wang W, Ma Y, Zeng Y, Dou D, Fan H, Song J, Yu X, Xin D, Du G, et al: Serum KIAA1199 is an advanced-stage prognostic biomarker and metastatic oncogene in cholangiocarcinoma. Aging (Albany NY) 12: 23761-23777, 2020.

4. Penner AS, Rock MJ, Kielty CM and Shipley JM: Microfibril-associated glycoprotein-2 interacts with fibrillin-1 and fibrillin-2 suggesting a role for MAGP-2 in elastic fiber assembly. J Biol Chem 277: 35044-35049, 2002.

5. CraftCS,BroekelmannTJandMechamRP:Microfibril-associated glycoproteins MAGP-1 and MAGP-2 in disease. Matrix Biol 71-72: 100-111, 2018.

6. Segade F, Suganuma N, Mychaleckyj JC and Mecham RP: The intracellular form of human MAGP1 elicits a complex and specific transcriptional response. Int J Biochem Cell Biol 39: 2303-2313, 2007.

7. Mecham RP and Gibson MA: The microfibril-associated glycoproteins (MAGPs) and the microfibrillar niche. Matrix Biol 47: 13-33, 2015.

8. Gong X, Dong T, Niu M, Liang X, Sun S, Zhang Y, Li Y and Li D: lncRNA LCPAT1 upregulation promotes breast cancer progression via enhancing MFAP2 transcription. Mol Ther Nucleic Acids 21: 804-813, 2020.

9. Dong SY, Chen H, Lin LZ, Jin L, Chen DX, Wang OC and Ye ZQ: MFAP2 is a potential diagnostic and prognostic biomarker that correlates with the progression of papillary thyroid cancer. Cancer Manag Res 12: 12557-12567, 2020.

10. Chen Z, Lv Y, Cao D, Li X and Li Y: Microfibril-associated protein 2 (MFAP2) potentiates invasion and migration of melanoma by EMT and Wnt/ $\beta$-catenin pathway. Med Sci Monit 26 : e923808, 2020.

11. Considine DPC, Jia G, Shu X, Schildkraut JM, Pharoah PDP, Zheng W and Kar SP; Ovarian Cancer Association Consortium: Genetically predicted circulating protein biomarkers and ovarian cancer risk. Gynecol Oncol 160: 506-513, 2021.

12. Yao LW, Wu LL, Zhang LH, Zhou W, Wu L, He K, Ren JC, Deng YC, Yang DM, Wang J, et al: MFAP2 is overexpressed in gastric cancer and promotes motility via the MFAP2/integrin

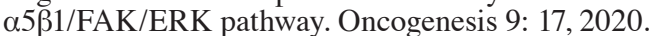

13. Sun T, Wang D, Ping Y, Sang Y, Dai Y, Wang Y, Liu Z, Duan X, Tao Z and Liu W: Integrated profiling identifies SLC5A6 and MFAP2 as novel diagnostic and prognostic biomarkers in gastric cancer patients. Int J Oncol 56: 460-469, 2020.

14. Zhu X, Cheng Y, Wu F, Sun H, Zheng W, Jiang W, Shi J, Ma S and Cao H: MFAP2 promotes the proliferation of cancer cells and is associated with a poor prognosis in hepatocellular carcinoma. Technol Cancer Res Treat 19: 1533033820977524, 2020
15. Zhai LL, Cai CY, Wu Y and Tang ZG: Correlation and prognostic significance of MMP-2 and TFPI-2 differential expression in pancreatic carcinoma. Int J Clin Exp Pathol 8: 682-691, 2015.

16. Zhai LL, Wu Y, Huang DW and Tang ZG: Increased matrix metalloproteinase-2 expression and reduced tissue factor pathway inhibitor-2 expression correlate with angiogenesis and early postoperative recurrence of pancreatic carcinoma. Am J Transl Res 7: 2412-2422, 2015.

17. Livak KJ and Schmittgen TD: Analysis of relative gene expression data using real-time quantitative PCR and the 2(-Delta Delta C(T)) method. Methods 25: 402-408, 2001.

18. Claesson-Welsh L and Welsh M: VEGFA and tumour angiogenesis. J Intern Med 273: 114-127, 2013.

19. Craft CS: MAGP1, the extracellular matrix, and metabolism. Adipocyte 4: 60-64, 2014.

20. Gibson MA, Hatzinikolas G, Kumaratilake JS, Sandberg LB, Nicholl JK, Sutherland GR and Cleary EG: Further characterization of proteins associated with elastic fiber microfibrils including the molecular cloning of MAGP-2 (MP25). J Biol Chem 271: 1096-1103, 1996.

21. Clarke AW and Weiss AS: Microfibril-associated glycoprotein-1 binding to tropoelastin: multiple binding sites and the role of divalent cations. Eur J Biochem 271: 3085-3090, 2004.

22. Ju S, Wang F, Wang Y and Ju S: CSN8 is a key regulator in hypoxia-induced epithelial-mesenchymal transition and dormancy of colorectal cancer cells. Mol Cancer 19: 168, 2020.

23. Jiang Z, Zhai X, Shi B, Luo D and Jin B: KIAA1199 overexpression is associated with abnormal expression of EMT markers and is a novel independent prognostic biomarker for hepatocellular carcinoma. Onco Targets Ther 11: 8341-8348, 2018.

24. Solanas G, Porta-de-la-Riva M, Agustí C, Casagolda D, Sánchez-Aguilera F, Larriba MJ, Pons F, Peiró S, Escrivà M, Muñoz A, et al: E-cadherin controls beta-catenin and NF-kappaB transcriptional activity in mesenchymal gene expression. J Cell Sci 121: 2224-2234, 2008.

25. Lamouille S, Xu J and Derynck R: Molecular mechanisms of epithelial-mesenchymal transition. Nat Rev Mol Cell Biol 15: 178-196, 2014.

26. Gonzalez DM and Medici D: Signaling mechanisms of the epithelial-mesenchymal transition. Sci Signal 7: re8, 2014.

27. Wendt MK, Tian M and Schiemann WP: Deconstructing the mechanisms and consequences of TGF- $\beta$-induced EMT during cancer progression. Cell Tissue Res 347: 85-101, 2012.

28. Willis BC and Borok Z: TGF-beta-induced EMT: Mechanisms and implications for fibrotic lung disease. Am J Physiol Lung Cell Mol Physiol 293: L525-L534, 2007.

29. Zhang Y, Ren H, Li J, Xue R, Liu H, Zhu Z, Pan C, Lin Y, Hu A, Gou P, et al: Elevated HMGB1 expression induced by hepatitis B virus $\mathrm{X}$ protein promotes epithelial-mesenchymal transition and angiogenesis through STAT3/miR-34a/NF- $\mathrm{KB}$ in primary liver cancer. Am J Cancer Res 11: 479-494, 2021.

30. Zhou W, Yang L, Nie L and Lin H: Unraveling the molecular mechanisms between inflammation and tumor angiogenesis. Am J Cancer Res 11: 301-317, 2021.

31. Jain S, Deore SV, Ghadi R, Chaudhari D, Kuche K and Katiyar SS: Tumor microenvironment responsive VEGF-antibody functionalized $\mathrm{pH}$ sensitive liposomes of docetaxel for augmented breast cancer therapy. Mater Sci Eng C Mater Biol Appl 121: 111832, 2021.

32. Kamiyama M and Augustin HG: Alternatively spliced form of angiopoietin-2 as a new vascular rheostat. Cancer Res 81: 35-37, 2021.

33. Varone E, Decio A, Chernorudskiy A, Minoli L, Brunelli L, Ioli F, Piotti A, Pastorelli R, Fratelli M, Gobbi M, et al: The ER stress response mediator ERO1 triggers cancer metastasis by favoring the angiogenic switch in hypoxic conditions. Oncogene 40: 1721-1736, 2021.

34. Quan L, Ohgaki R, Hara S, Okuda S, Wei L, Okanishi H, Nagamori S, Endou H and Kanai Y: Amino acid transporter LAT1 in tumor-associated vascular endothelium promotes angiogenesis by regulating cell proliferation and VEGF-A-dependent mTORC1 activation. J Exp Clin Cancer Res 39: 266, 2020.

This work is licensed under a Creative Commons Attribution-NonCommercial-NoDerivatives 4.0 International (CC BY-NC-ND 4.0) License. 